\title{
Alergia a Medicamentos Reportada em Crianças que Frequentam Infantários
}

\author{
Reported Drug Allergy among Children Attending Day Care Centers
}

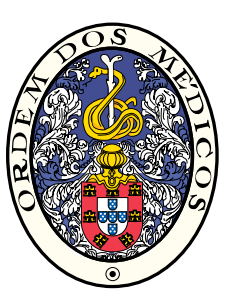

\author{
Pedro MARTINS ${ }^{1,2,4}$, Joana BELO ${ }^{1,2}$, João MARQUES ${ }^{1,2}$, Ana Luísa PAPOILA ${ }^{3,4}$, lolanda CAIRES ${ }^{1}$, José ARAÚJO- \\ MARTINS ${ }^{1}$, Catarina PEDRO ${ }^{1}$, José ROSADO-PINTO ${ }^{5}$, Daniel VIRELLA ${ }^{4}$, Paula LEIRIA-PINTO ${ }^{1,2}$, Nuno NEUPARTH ${ }^{1,2}$ \\ Acta Med Port 2014 Jul-Aug;27(4):444-449
}

\section{RESUMO}

Introdução: A prevalência de alergia a fármacos na população geral não se encontra devidamente caraterizada, existindo poucos estudos publicados que tenham abordado esta situação em crianças com idades inferior a seis anos de idade. Este estudo tem como objetivo principal estimar a prevalência de alergia a medicamentos reportada pelos pais de crianças de infantários de Lisboa e do Porto. Material e Métodos: No âmbito da Fase II do projeto "ENVIRH - Ambiente e Saúde em Creches e Infantários" foi aplicado um questionário sobre alergia a medicamentos aos pais das crianças, recrutadas por amostragem aleatória estratificada dos infantários.

Resultados: Foram analisados 1169 questionários, 52,5\% de rapazes. A idade média foi de 3,5 $\pm 1,5$ anos. A prevalência de alergia a medicamentos reportada foi de 4,1\% (IC 95\%: 3,0 - 5,2\%). Os fármacos mais referidos foram os antibióticos (em 27 reações) e os AINEs (em seis reações). Na análise multivariável, a alergia a medicamentos reportada associou-se diretamente com a idade da criança (OR 1,19; IC 95\% 1,01 - 1,41) e com a referência a alergia alimentar (OR 3,19; IC95\% 1,41 - 7,19) e inversamente com o nível de escolaridade dos pais (OR 0,25; IC95\% 0,10 - 0,59).

Discussão: Apesar das limitações do estudo, os resultados encontram-se de acordo com o reportado por outros autores e sugerem que a prevalência reportada de alergia a medicamentos seja elevada no grupo etário estudado.

Conclusão: Torna-se necessário que situações de alergia a medicamentos reportadas pelos pais sejam devidamente estudadas, no sentido de evitar evicções desnecessárias que possam condicionar opções terapêuticas em futuras situações de doença.

Palavras-chave: Creches; Criança; Hipersensibilidade a Medicamentos; Prevalência; Portugal.

\section{ABSTRACT}

Introduction: Data about drug allergy prevalence in the general population, particularly in children, are lacking. This study aimed to estimate the prevalence of parent-reported drug allergy, in children attending day care centers in Lisbon and Oporto.

Material and Methods: In Phase II of the "ENVIRH study - Environment and Health in Children Day Care Centers", a health questionnaire which included questions about drug allergies was administered to children by stratified, random sampling of day care centers.

Results: The final analysis included 1,169 questionnaires, $52.5 \%$ from boys. The mean age was $3.5 \pm 1.5$ years. The prevalence of reported drug allergy was $4.1 \%(95 \% \mathrm{Cl}: 3.0-5.2 \%)$. The most frequently reported drugs were antibiotics (27 cases) and NSAIDs (in 6 cases). In the multivariate analysis, reported drug allergy was directly associated with age (OR $1.19 ; 95 \% \mathrm{Cl} 1.01-1.41)$ and reported food allergy (OR $3.19 ; 95 \% \mathrm{Cl} 1.41-7.19)$. It was inversely associated with the level of parental education $(\mathrm{OR} 0.25 ; 95 \% \mathrm{Cl}$ $0.10-0.59)$.

Discussion: Even though the limitations of the study our results are in accordance with those reported by previous authors and suggest that there is a high prevalence of reported drug allergy in the considered age group.

Conclusion: A correct assessment of these situations is needed in order to avoid unnecessary drug evictions.

Keywords: Child Day Care Centers; Child; Drug Hypersensitivity; Prevalence; Portugal.

\section{INTRODUÇÃO}

As reações adversas a medicamentos (RAM) constituem um grave problema de saúde pública, estimando-se que afetem $7 \%$ da população geral e $20 \%$ dos doentes hospitalizados. ${ }^{1}$ Têm também sido apontadas como causas de morte relevantes quer em adultos, quer em crianças. ${ }^{2}$

A Organização Mundial de Saúde define RAM como qualquer resposta prejudicial, não intencional e indesejável, a um fármaco administrado em doses normalmente usadas em seres humanos para a profilaxia, diagnóstico ou terapia. ${ }^{3,4}$

As RAM podem ser classificadas em vários subtipos. ${ }^{5}$
As RAM de tipo B (cerca de $15 \%),{ }^{3}$ que atingem somente indivíduos suscetíveis e são independentes da dose, incluem as reações de hipersensibilidade.

As reações de hipersensibilidade alérgicas definem-se como reações adversas com um mecanismo imunológico subjacente, que surgem na sequência da administração de um medicamento. ${ }^{6}$ Estima-se que até $1 / 3$ das RAM em doentes hospitalizados possa ser de etiologia alérgica. ${ }^{3}$

A prevalência de alergia a fármacos na população geral não se encontra devidamente caraterizada. Estudos transversais referem que cerca de $7 \%$ da população reporta

1. Centro de Estudos de Doenças Crónicas. Faculdade de Ciências Médicas. Universidade Nova de Lisboa. Lisboa. Portugal.

2. Serviço de Imunoalergologia. Hospital de Dona Estefânia. Centro Hospitalar Lisboa Central. Lisboa. Portugal.

3. Departamento de Bioestatística e Informática. Centro de Estudos Anglísticos. Faculdade de Ciências Médicas. Universidade Nova de Lisboa. Lisboa. Portugal.

4. Centro de Investigação. Hospital de Dona Estefânia. Centro Hospitalar Lisboa Central. Lisboa. Portugal.

5. Serviço de Imunoalergologia. Hospital da Luz. Lisboa. Portugal.

Recebido: 27 de Agosto de 2013 - Aceite: 03 de Dezembro de 2013 | Copyright @ Ordem dos Médicos 2014 
alergia a medicamentos. ${ }^{1,7}$ Existem poucos estudos publicados que tenham caraterizado esta situação em crianças com idade inferior a seis anos de idade. Pensa-se que a prevalência de alergia a fármacos na criança seja inferior à dos adultos, ${ }^{3}$ mas a grande maioria dos estudos tem por base dados hospitalares. Os dados disponíveis apontam para que os antibióticos lactâmicos sejam a causa mais comum de alergia a medicamentos em idade pediátrica. ${ }^{8}$

$\mathrm{Na}$ prática clínica, após uma reação adversa a um medicamento, os doentes são frequentemente classificados como sendo alérgicos, mesmo sem qualquer investigação. Contudo, muitas das reações adversas associadas à toma de um medicamento serão de etiologia infeciosa e não verdadeiras alergias. ${ }^{1,9}$ Sabe-se que quando a história de alergia a um medicamento é complementada pela realização de testes cutâneos ou através de prova de provocação oral, a grande maioria tolerará o medicamento sem qualquer reação. $8,10,11$

Este estudo teve como objetivo principal estimar a prevalência de alergia a medicamentos reportada numa amostra de crianças que frequenta infantários de Lisboa e do Porto, procurando ainda identificar variáveis associadas com a alergia reportada.

\section{MATERIAL E MÉTODOS}

Na Fase I do projeto "ENVIRH - Ambiente e Saúde em Creches e Infantários", foi estudada uma amostra aleatória estratificada por freguesia de 45 infantários, das cidades de Lisboa e do Porto, e das crianças que os frequentavam. $\mathrm{Na}$ Fase II, da amostra inicial, foram recrutados 19 infantários (11 em Lisboa e oito no Porto), selecionados por análise de clusters que teve em consideração parâmetros da qualidade do ar interior. Em ambas as fases, de entre as crianças que frequentavam os infantários seleccionados, foram recrutadas amostras de conveniência, para inquirição, e subamostras, para outros estudos. O presente estudo reporta resultados da Fase II.

Em Fevereiro de 2011, um questionário que incluía perguntas da versão portuguesa do International Study of Asthma and Allergies in Childhood (ISAAC) $)^{12}$ e questões sobre alergia a medicamentos, foi distribuído pelas educadoras dos infantários selecionados aos pais das crianças que os frequentavam. Se à questão ' $O$ seu filho tem alergia a algum medicamento?' fosse dada resposta afirmativa, era solicitada a identificação do medicamento implicado, através de uma pergunta de escolha múltipla.

Os pais que reportaram que o filho tinha alergia a medicamentos foram contatados para melhor caraterização da reação, tendo sido aplicado novo questionário. De acordo com a história clínica, as reações foram classificadas como imediatas (se ocorreram na primeira hora após a toma) ou não imediatas (se surgiram após a primeira hora). ${ }^{13} \mathrm{~A}$ classificação de Ring e Messmer foi utilizada para as reações imediatas. ${ }^{14}$ As não imediatas foram classificadas como ligeiras (se não necessitaram de tratamento), moderadas (se necessitaram de tratamento, houve boa resposta e não foi necessária hospitalização) e graves (se necessitou de hospitalização ou houve risco de vida). Anafilaxia foi definida de acordo com as recomendações existentes. , $^{6,15}$

Efetuou-se uma análise exploratória das variáveis de interesse, sendo a variável resposta a referência à existência de alergia a medicamentos. As variáveis independentes foram o género, a idade, a existência de diagnóstico médico de eczema, a referência a alergia alimentar, a história familiar de asma ou rinite alérgica (diagnóstico reportado) e o nível de escolaridade dos pais (dicotomizada em pelo menos um dos pais ter ou não licenciatura concluída). Utilizaram-se modelos de regressão logística para identificar fatores associados com alergia a medicamentos reportada. $\mathrm{Na}$ análise multivariável foram incluídas todas as variáveis que na análise univariável apresentaram associação com a variável resposta, com um valor $p<0,25$. São apresentados os odds ratio (OR) brutos e ajustados, com os respetivos intervalos de confiança a 95\% (IC 95\%). O nível de significância considerado foi $\alpha=0,05 .{ }^{16} \mathrm{~A}$ análise dos dados foi efetuada através do programa SPSS 20.0 (Statistical Package for the Social Sciences. IBM, New York, EUA).

O projeto ENVIRH foi aprovado pela Comissão de Ética da Faculdade de Ciências Médicas da Universidade Nova de Lisboa. Os pais das crianças participantes deram consentimento informado por escrito.

\section{RESULTADOS}

Dos 2287 questionários entregues para preenchimento (1 415 em Lisboa e 872 no Porto), foram devolvidos 1225. Destes, incluíram-se na análise os 1169 (51\%) com resposta à pergunta sobre alergia a medicamentos. A proporção de inquéritos analisados face ao número total entregue, foi de $49 \%$ em Lisboa e de $52 \%$ no Porto $(p=0,026)$.

Não foram encontradas diferenças significativas do género entre as crianças que responderam ao questionário e as não respondentes ( $p=0,700)$. Também não se observaram diferenças relativamente às proporções de crianças com idade inferior e superior a três anos de idade $(p=1,00)$, entre as crianças que responderam ao questionário e as não respondentes.

A amostra avaliada foi composta por $52,5 \%$ de rapazes; a idade média foi de 3,5 $\pm 1,5$ anos. A escolaridade dos pais das crianças era pelo menos licenciatura em 32,4\% (40,6\% em Lisboa e $20,1 \%$ no Porto, $p<0,001)$. As caraterísticas dos elementos da amostra são apresentadas na Tabela 1.

A alergia a medicamentos foi reportada em $4,1 \%$ das crianças (IC 95\%: 3,0 - 5,2), sendo a prevalência reportada significativamente superior na amostra da cidade do Porto (Lisboa 2,1\%, IC 95\%: 1,3 - 3,5; e Porto 6,9\%, IC 95\%: 5.0 - 9,6 p<0,001).

Os fármacos mais frequentemente referidos como implicados na reação foram os antibióticos (lactâmicos em 14 casos, macrólidos em dois; em 11 o antibiótico não foi especificado), anti-infamatórios não esteróides - AINEs (ibuprofeno em cinco casos e ácido acetilsalicílico em um) e os anti-histamínicos (quatro casos). Foram ainda reportadas reações para colírios (três casos), acetilcisteina (um caso) e amilase (um caso). Nos restantes seis não foi especificado 


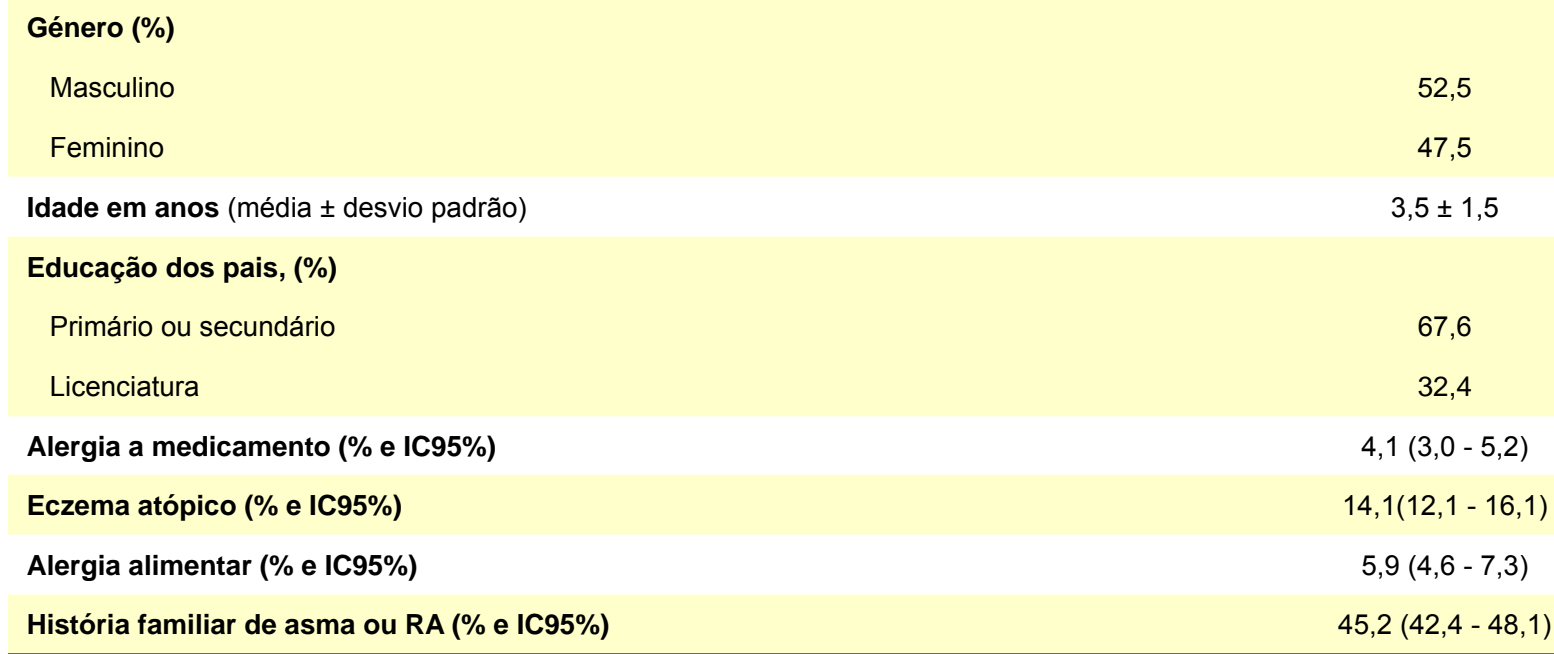

RA: rinite alérgica; IC95\%: intervalo de confiança de $95 \%$

Tabela 2 - Caraterísticas das reações a medicamentos reportadas $(n=34)$

\begin{tabular}{|c|c|}
\hline & TOTAL \\
\hline Idade quando ocorreu a reação (média \pm DP) & $2,2 \pm 1,4$ anos \\
\hline \multicolumn{2}{|l|}{ Cronologia } \\
\hline Imediata & 3 \\
\hline Não imediata & 31 \\
\hline \multicolumn{2}{|l|}{ Classificação (gravidade) } \\
\hline \multicolumn{2}{|l|}{ Imediata } \\
\hline Grau I & 2 \\
\hline Grau II & 0 \\
\hline Grau III & 1 \\
\hline Grau IV & 0 \\
\hline \multicolumn{2}{|l|}{ Não imediata } \\
\hline Ligeira & 7 \\
\hline Moderada & 24 \\
\hline Grave & 0 \\
\hline \multicolumn{2}{|l|}{ Apresentação clínica da reação } \\
\hline Mucocutânea isolada & 26 \\
\hline Gastrointestinal isolada & 4 \\
\hline Mucocutânea e gastrointestinal & 3 \\
\hline Mucocutânea, gastrointestinal e respiratória & 1 \\
\hline \multicolumn{2}{|l|}{ História familiar de alergia a medicamentos } \\
\hline Sim & 10 \\
\hline Não & 24 \\
\hline
\end{tabular}

DP: desvio padrão

o medicamento implicado.

Foram obtidos mais detalhes sobre a reação adversa ao medicamento em 34 das 48 reações (Tabela 2). Nestas, a média de idades no momento da reação foi de 2,2 $\pm 1,4$ anos. Em apenas três reações a apresentação foi imediata (duas de grau I e outra de grau III). A totalidade das 31 reações não imediatas caraterizadas foi ligeira (7) ou moderada (24).

A manifestação clínica mais comum foi a de carácter mucocutâneo isolado (em 26 reações), seguida da 
Tabela 3 - Associações entre a referência a alergia a medicamentos e outras variáveis estudadas

\begin{tabular}{lcc}
\hline & \multicolumn{1}{c}{$\begin{array}{c}\text { Referência a alergia a medicamentos } \\
\text { Odds ratio brutos } \\
\text { (IC } 95 \%)\end{array}$} & $\begin{array}{c}\text { Odds ratio ajustados } \\
\text { (IC 95\%) }\end{array}$ \\
\hline Género (feminino) & $0,78(0,44-1,41), p=0,411$ & $*$ \\
Idade (anos) & $1,23,(1,06-1,46), p=0,009$ & $1,19(1,01-1,41), p=0,038$ \\
$\begin{array}{l}\text { Nível de escolaridade dos pais } \\
\text { ter licenciatura) }\end{array}$ & $0,24(0,10-0,56), p=0,001$ & $0,25(0,10-0,59), p=0,002$ \\
Eczema atópico & $0,77(0,30-1,98), p=0,580$ & $*$ \\
Referência a alergia alimentar & $3,31(1,48-7,40), p=0,003$ & $3,19(1,41-7,19), p=0,005$ \\
História familiar de asma ou RA & $0,86(0,48-1,55), p=0,619$ & $*$
\end{tabular}

Foram incluídas no modelo múltiplo final as seguintes variáveis: idade, nível de escolaridade dos pais, existência de alergia alimentar reportada; RA; rinite alérgica; IC95\%: intervalo de confiança de $95 \%$; * variável não selecionada para a análise multivariada por $p$ na análise univariável > 0,25.

gastrointestinal isolada (em quatro reações) e de sintomas mucocutâneos e gastrointestinais (em três reações). Num caso foi reportado uma reação com sintomas mucocutâneos, gastrointestinais e respiratórios (Tabela 2), tendo sido esta de carácter imediato, cumprindo de uma forma clara os critérios de anafilaxia. As outras duas reações imediatas reportadas tiveram apresentação exclusivamente mucocutânea.

Os pais de dez das crianças reportaram também serem alérgicos a medicamentos. Somente duas crianças tinham sido avaliadas em consulta de Imunoalergologia na sequência da reação, confirmando-se num dos casos alergia a lactâmicos.

Quer na análise univariável quer na multivariável, os fatores que se associaram diretamente com a referência a alergia a medicamentos foram a idade da criança e a referência a alergia alimentar (Tabela 3 ). A educação dos pais associou-se inversamente com a referência a alergia a medicamentos.

\section{DISCUSSÃO}

No presente estudo foi encontrada uma prevalência reportada de alergia a medicamentos de $4,1 \%$, tendo sido os antibióticos e os AINEs os agentes mais frequentemente referidos, provavelmente reflexo das caraterísticas de prescrição nesta faixa etária. Este achado está de acordo com o reportado por outros autores..$^{8,17-19}$

Num estudo efetuado na Turquia, ${ }^{20}$ numa amostra de 2 855 crianças dos seis aos nove anos, foi encontrada uma prevalência reportada de $2,8 \%$. O estudo de Rebelo Gomes et al $^{8}$ numa amostra de 1426 crianças com uma idade média de 7,3 $\pm 4,3$ anos, recrutadas em consultas externas de diversas especialidades da cidade do Porto, encontrou uma prevalência reportada de alergia a medicamentos de $6,0 \%$. Após investigação, foi excluída a existência de alergia a medicamentos em $94 \%$ destes casos. Tal poderá ser explicado pelo facto do quadro clínico ser frequentemente expressão de uma doença infeciosa., ${ }^{9,21}$
As RAMs constituem um importante problema de saúde pública, sendo consideradas como umas das principais causas de morte nos Estados Unidos da América, ${ }^{2}$ mesmo quando os medicamentos são utilizados de acordo com a posologia e a indicação terapêutica. A maioria destas reações $(80 \%)$ é do tipo $A$, sendo cerca de $15 \%$ do tipo $B,^{3}$ categoria onde se encontram as reações de hipersensibilidade.

De acordo com uma meta-análise, ${ }^{22}$ a incidência de RAMs em crianças hospitalizadas, considerando todos os tipos de gravidade, foi de $10,9 \%$. As incidências de reações de maior gravidade e reações fatais foram de $2,1 \%$ e $0,19 \%$, respetivamente.

Existem poucos estudos publicados sobre a prevalência de alergia a medicamentos na criança, ${ }^{8,10,23}$ e em particular em crianças com idade inferior a seis anos. Contudo, a literatura existente sugere que, à semelhança do que acontece noutras faixas etárias, ${ }^{24}$ somente uma minoria daqueles que reportam alergia serão verdadeiramente alérgicos.

$\mathrm{Na}$ amostra do estudo apresentado, somente uma minoria das crianças (2/34) tinha sido referenciada para consulta especializada, para esclarecer a reação, semelhante ao observado por outros autores. ${ }^{23}$ Partindo do pressuposto de que a grande maioria das RAMs reportadas não são de causa alérgica, tal como foi já documentado pelos autores ${ }^{11}$ para o caso dos antibióticos betalactâmicos, torna-se necessário promover a investigação destas situações. ${ }^{25}$ Está referido que, nas reações com apresentação predominantemente cutânea, uma avaliação exclusivamente clínica tende a sobrestimar o diagnóstico de alergia a medicamentos. ${ }^{26}$ Acresce ainda que a grande maioria das reações reportadas são de gravidade ligeira ou moderada, em que o risco de investigação com eventual reexposição controlada ao fármaco poderá ser inferior ao da evicção desnecessária.

A orientação da situação para investigação poderá ser feita pelo médico que observe a reação no serviço de urgência ou pelo médico assistente da criança. 
Em termos de métodos de diagnóstico, no âmbito de uma consulta de Imunoalergologia, podem ser efetuados testes cutâneos para os medicamentos que apresentam concentrações máximas não irritativas devidamente validadas, como no caso dos antibióticos lactâmicos. A literatura sugere-nos que os doentes com história de reação à penicilina, se tiverem testes cutâneos negativos, terão um risco sobreponível ao da população geral de sofrer uma reação numa futura exposição à penicilina. ${ }^{27}$

À semelhança do descrito para o adulto, ${ }^{28}$ no presente estudo, a alergia a medicamentos associou-se diretamente com a idade da criança. Tal poderá ser explicado por uma eventual imaturidade imunológica ou por uma menor exposição prévia a fármacos, nas crianças mais novas. ${ }^{22}$

A associação encontrada entre alergia a medicamentos e alergia alimentar está de acordo com estudos que demonstram que a alergia alimentar pode associar-se a maior possibilidade de ter alergia a medicamentos devidamente confirmada. ${ }^{11}$ Várias causas poderão ser apontadas para este facto, como fatores imunológico ${ }^{29}$ ou psicossociais ${ }^{30}$ que aumentarão a perceção da doença.

Os indicadores de atopia utilizados (eczema atópico e história familiar de alergia respiratória) não se associaram com maior possibilidade de reportar alergia a medicamentos neste estudo. Não é ainda claro o papel da atopia, pensando-se atualmente que não constitua um fator de risco para alergia a medicamentos ${ }^{31}$ mas que se possa associar com reações de maior gravidade.

O baixo nível de instrução é um conhecido fator de risco para prevalência aumentada da generalidade das doenças. ${ }^{32}$ Encontram-se no entanto descritas algumas exceções, como são os casos da asma e da rinite alérgica. Esta associação entre aumento de risco de doença e baixo nível de escolaridade deve-se ao facto de a educação ser um indicador do nível socio-económico. Encontrámos uma associação inversa entre o nível de escolaridade dos pais e a referência a alergia a medicamentos. Para isso poderá contribuir a maior exposição a medicamentos nos filhos de pais com menor nível de instrução, por ficarem doentes mais frequentemente. Por outro lado, a interpretação do termo 'alergia' pode diferir entre pais com diferentes níveis de instrução. Estes aspetos podem contribuir para explicar a diferença de prevalência reportada de alergia a medicamentos encontrada nas duas cidades, dado o menor grau de escolaridade dos pais das crianças do Porto na nossa amostra.

A estimativa apresentada de prevalência reportada de reações alérgicas a medicamentos baseia-se numa amostra de conveniência de crianças, determinada pela aceitação dos pais em participar no estudo, recrutadas por frequentarem infantários seleccionados por uma amostragem desenhada para outro fim. Não há evidência de que os critérios utilizados na amostragem dos infantários, estratificada (freguesias) e por clusters (parâmetros ambien- tais), possam enviesar a amostragem de crianças que frequentam infantários para estimar a prevalência reportada de reações alérgicas a medicamentos. Não é possível no entanto, garantir que uma amostra recrutada em infantários seja representativa da globalidade da população neste grupo etário, nem que a amostra obtida por consentimento parental seja representativa das crianças que frequentam esses infantários. É possível que haja maior adesão à participação nestes estudos entre as famílias com história de doenças atópicas ou respiratórias, o que poderia sobrevalorizar a estimativa da prevalência do problema. Por outro lado, a amostragem de conveniência permitiu recrutar uma amostra muito maior do que as 850 crianças que seriam necessárias recrutar aleatoriamente para estimar uma prevalência de $4 \%$, assumindo um erro de $\pm 1 \%$, com $95 \%$ de confiança.

Outra limitação deste estudo advém do facto da existência de alergia a medicamentos ter sido estabelecida com base em questionário, que tende a sobrestimar a verdadeira prevalência. Também não foi possível caraterizar todas as reações reportadas de forma detalhada. Contudo, os resultados obtidos são um contributo para a quantificação do problema das reações alérgicas a medicamentos reportadas crianças de idade inferior a seis anos, e permitem estimar que uma proporção significativa de crianças poderá ser privada de medicamentos, designadamente antibióticos e AINEs, por estarem classificadas incorretamente como alérgicas.

\section{CONCLUSÃO}

Neste estudo observamos uma prevalência de $4 \%$ de crianças com idade inferior a seis anos, que se encontra rotulada como alérgica a medicamentos. Tendo em conta que a grande maioria não é verdadeiramente alérgica, torna-se necessário que estas situações sejam devidamente estudadas, para evitar evicções desnecessárias que possam condicionar opções terapêuticas em futuras situações de doença.

\section{AGRADECIMENTOS}

A equipa de investigadores agradece aos pais e às crianças participantes, bem como aos educadores, aos responsáveis dos infantários e a todas as autoridades que tornaram este estudo possível.

\section{CONFLITOS DE INTERESSE}

Os autores declaram a inexistência de conflitos de interesse na realização do presente trabalho.

\section{FONTES DE FINANCIAMENTO}

Fundação para a Ciência e Tecnologia - ENVIRH Project PTDC/SAU-ESA/100275/2008 e PEst-OE/MAT/ UI0006/2011. 


\section{REFERÊNCIAS}

1. Gomes ER, Demoly P. Epidemiology of hypersensitivity drug reactions. Curr Opin Allergy Clin Immunol. 2005;5:309-16.

2. Lazarou J, Pomeranz BH, Corey PN. Incidence of adverse drug reactions in hospitalized patients: a meta-analysis of prospective studies. JAMA. 1998;279:1200-5.

3. Thong BY, Tan TC. Epidemiology and risk factors for drug allergy. Br J Clin Pharmacol. 2011;71:684-700.

4. Joint Task Force on Practice Parameters, American Academy of Allergy, Asthma and Immunology, American College of Allergy, Asthma and Immunology, Joint Council of Allergy, Asthma and Immunology. Drug allergy: an updated practice parameter. Ann Allergy Asthma Immunol. 2010;105:259-73.

5. Edwards IR, Aronson JK. Adverse drug reactions: definitions, diagnosis, and management. Lancet. 2000;356:1255-9.

6. Johansson SG, Bieber T, Dahl R, Friedmann PS, Lanier BQ, Lockey $\mathrm{RF}$, et al. Revised nomenclature for allergy for global use: Report of the Nomenclature Review Committee of the World Allergy Organization, October 2003. J Allergy Clin Immunol. 2004;113:832-6.

7. Falcao H, Lunet N, Gomes E, Cunha L, Barros H. Drug allergy in university students from Porto, Portugal. Allergy. 2003;58:1210.

8. Rebelo Gomes E, Fonseca J, Araujo L, Demoly P. Drug allergy claims in children: from self-reporting to confirmed diagnosis. Clin Exp Allergy. 2008;38:191-8.

9. Romano A, Quaratino D, Papa G, Di Fonso M, Venuti A. Aminopenicillin allergy. Arch Dis Child. 1997;76:513-7.

10. Gomes E, Cardoso MF, Praca F, Gomes L, Marino E, Demoly P. Selfreported drug allergy in a general adult Portuguese population. Clin Exp Allergy. 2004;34:1597-601.

11. Chambel M, Martins P, Silva I, Palma-Carlos S, Romeira AM, Leiria Pinto $P$. Drug provocation tests to betalactam antibiotics: experience in a paediatric setting. Allergol Immunopathol. 2010;38:300-6

12. Rosado-Pinto J. ISAAC (International Study of Asthma and Allergiesin Childhood) 20 Anos em Portugal. Acta Pediatr Port. 2011;42:S2.

13. Romano A, Blanca M, Torres MJ, Bircher A, Aberer W, Brockow K, et al. Diagnosis of nonimmediate reactions to beta-lactam antibiotics. Allergy. 2004;59:1153-60.

14. Ring J, Messmer K. Incidence and severity of anaphylactoid reactions to colloid volume substitutes. Lancet. 1977;1:466-9.

15. Sampson HA, Munoz-Furlong A, Bock SA, Schmitt C, Bass R, Chowdhury BA, et al. Symposium on the definition and management of anaphylaxis: summary report. J Allergy Clin Immunol. 2005;115:584-91.

16. Altman D. Practical statistical for medical research. London: Chapman \& Hall; 1990

17. Caubet JC, Eigenmann PA. Diagnostic issues in pediatric drug allergy.
Curr Opin Allergy Clin Immunol. 2012;12:341-7.

18. Gamboa PM. The epidemiology of drug allergy-related consultations in Spanish allergology services: Alergologica-2005. J Investig Allergol Clin Immunol. 2009;19:S45-50.

19. Ribeiro-Vaz I, Marques J, Demoly P, Polonia J, Gomes ER. Druginduced anaphylaxis: a decade review of reporting to the Portuguese Pharmacovigilance Authority. Eur J Clin Pharmacol. 2013;69:673-81.

20. Orhan F, Karakas T, Cakir M, Akkol N, Bahat E, Sonmez FM, et al. Parental-reported drug allergy in 6- to 9-yr-old urban schoolchildren. Pediatr Allergy Immunol. 2008;19:82-5.

21. Demoly P, Gomes ER. Drug hypersensitivities: definition, epidemiology and risk factors. Eur Ann Allergy Clin Immunol. 2005;37:202-6.

22. Impicciatore $P$, Choonara I, Clarkson A, Provasi D, Pandolfini C, Bonati $\mathrm{M}$. Incidence of adverse drug reactions in paediatric in/out-patients: a systematic review and meta-analysis of prospective studies. $\mathrm{Br} \mathrm{J} \mathrm{Clin}$ Pharmacol. 2001;52:77-83.

23. Tan VA, Gerez IF, Van Bever HP. Prevalence of drug allergy in Singaporean children. Singapore Med J. 2009;50:1158-61.

24. Messaad D, Sahla H, Benahmed S, Godard P, Bousquet J, Demoly P. Drug provocation tests in patients with a history suggesting an immediate drug hypersensitivity reaction. Ann Intern Med. 2004;140:1001-6.

25. Langley J, Halperin S. Allergy to antibiotics in children: perception versus reality. Can J Infect Dis. 2002;13:160-3.

26. Heinzerling LM, Tomsitz D, Anliker MD. Is drug allergy less prevalent than previously assumed? A 5-year analysis. $\mathrm{Br} J$ Dermatol. 2012;166:107-14.

27. del Real GA, Rose ME, Ramirez-Atamoros MT, Hammel J, Gordon SM Arroliga AC, et al. Penicillin skin testing in patients with a history of betalactam allergy. Ann Allergy Asthma Immunol. 2007;98:355-9.

28. Demoly P, Bousquet J. Epidemiology of drug allergy. Curr Opin Allergy Clin Immunol. 2001;1:305-10.

29. Selmi C, Crotti C, Meroni PL. Less travelled roads in clinical immunology and allergy: drug reactions and the environmental influence. Clin Rev Allergy Immunol. 2013;45:1-5.

30. Frostholm L, Fink P, Christensen KS, Toft T, Oernboel E, Olesen F, et al. The patients' illness perceptions and the use of primary health care Psychosom Med. 2005;67:997-1005.

31. Haddi E, Charpin D, Tafforeau M, Kulling G, Lanteaume A, Kleisbauer JP, et al. Atopy and systemic reactions to drugs. Allergy. 1990;45:236-9.

32. Dalstra JA, Kunst AE, Borrell C, Breeze E, Cambois E, Costa G, et al. Socioeconomic differences in the prevalence of common chronic diseases: an overview of eight European countries. Int J Epidemiol. 2005;34:316-26 
Pedro MARTINS, Joana BELO, João MARQUES, Ana Luísa PAPOILA, Iolanda CAIRES, José ARAÚJO-MARTINS, Catarina PEDRO, José ROSADO-PINTO, Daniel VIRELLA, Paula LEIRIA-PINTO, Nuno NEUPARTH

\section{Alergia a Medicamentos Reportada em Crianças que Frequentam Infantários \\ Acta Med Port 2014:27:444-449}

Publicado pela Acta Médica Portuguesa, a Revista Científica da Ordem dos Médicos

Av. Almirante Gago Coutinho, 151

1749-084 Lisboa, Portugal.

Tel: +351218428215

E-mail: submissao@actamedicaportuguesa.com

www.actamedicaportuguesa.com

ISSN:0870-399X | e-ISSN: 1646-0758

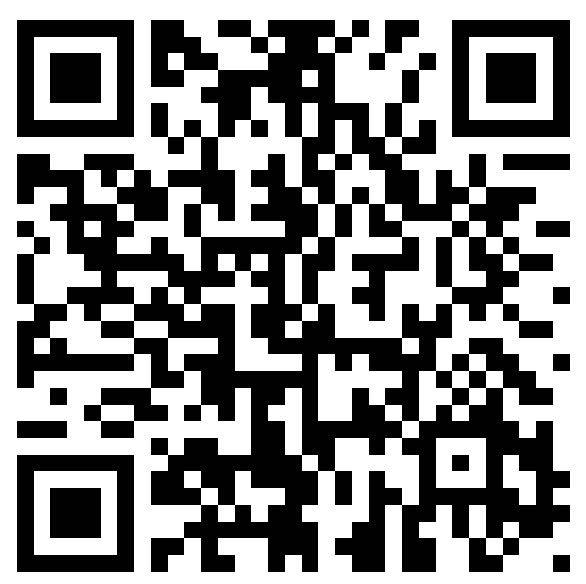

